\title{
ON THE HEISENBERG SUB-LORENTZIAN METRIC ON $\mathbb{R}^{3}$
}

\author{
MAREK GROCHOWSKI \\ Faculty of Mathematics and Sciences, Cardinal Stefan Wyszyński University \\ ul. Dewajtis 5, 01-815 Warszawa, Poland \\ E-mail: mgrochow@impan.gov.pl
}

\begin{abstract}
In this paper we study properties of the Heisenberg sub-Lorentzian metric on $\mathbb{R}^{3}$. We compute the conjugate locus of the origin, and prove that the sub-Lorentzian distance in this case is differentiable on some open set. We also prove the existence of regular non-Hamiltonian geodesics, a phenomenon which does not occur in the sub-Riemannian case.
\end{abstract}

\section{Introduction}

1.1. Statement of the results. There are many papers on the sub-Riemannian geometry, especially those treating a contact case in $\mathbb{R}^{3}$ (see for instance [1], [2] and their reference sections; see also [5]). On the contrary, the sub-Lorentzian geometry is almost not known, and [6] seems to be the first paper devoted to this subject.

By a sub-Lorentzian manifold we mean a triple $(M, H, h)$, where $M$ is a smooth (i.e. of class $C^{\infty}$ ) manifold, $H$ is a smooth bracket generating distribution of constant rank (i.e. $\operatorname{dim} H_{p}=k$ is independent of a point $p$; of course only the case $1<\operatorname{dim} H_{p}<\operatorname{dim} M$ is interesting), and $h$ is a Lorentzian metric on $H$. We say that $(M, H, h)$ is contact if $H$ is a contact distribution. Below in Section 1.2 we present, without proofs, a short review of basic notions and facts on sub-Lorentzian manifolds.

In this paper we deal with the simplest contact sub-Lorentzian manifold; it can be obtained as follows. Let $M=\mathbb{R}^{3}$ with standard coordinates $x, y, z$, and let

$$
\omega=d z-(y d x-x d y) / 2
$$

be the standard contact form on $\mathbb{R}^{3}$. We take $H=\operatorname{ker} \omega$. Next set

$$
X=\partial / \partial x+(1 / 2) y \partial / \partial z, \quad Y=\partial / \partial y-(1 / 2) x \partial / \partial z
$$

2000 Mathematics Subject Classification: 53C50.

Key words and phrases: sub-Lorentzian manifolds, geodesics.

The paper is in final form and no version of it will be published elsewhere. 
clearly $X$ and $Y$ form a global basis of $H$. Now define a Lorentzian metric $h$ on $H$ by setting

$$
h(X, X)=-1, \quad h(Y, Y)=1, \quad h(X, Y)=0,
$$

and take $X$ to be a time orientation. By analogy to the sub-Riemannian case we will call $(H, h)$ the Heisenberg sub-Lorentzian metric on $\mathbb{R}^{3}$.

In Section 2.1 we obtain equations of Hamiltonian geodesics for the Heisenberg metric, which can be explicitly integrated. In particular we prove that through the origin there pass only two null Hamiltonian geodesics, from which we find that in the contact subLorentzian geometry the exponential mapping with the pole, say, $p_{0}$ is not onto any neighbourhood of $p_{0}$ (recall that in the contact sub-Riemannian geometry the exponential mapping is always onto a certain neighbourhood of its pole).

In Section 2.2 we study the Heisenberg exponential mapping; we also compute the conjugate locus of the origin, which happens to coincide with the two null Hamiltonian geodesics passing through this point.

In Section 2.3 we study differential properties of the Heisenberg sub-Lorentzian distance function. In [6], because of some technical problems, we introduced two additional assumptions on $(M, H, h)$ (i.e. the condition (A) and points of finite type) to be able to prove differentiability of the general sub-Lorentzian distance function. It turns out that we do not need such assumptions in the Heisenberg case, and the distance is smooth on some open set.

In the sub-Riemannian geometry all geodesics can be of two types: Hamiltonian geodesics, which can be regular or singular curves, and so-called strictly abnormal extremals, which are necessarily singular. In particular each geodesic which is a regular curve is Hamiltonian. In Section 2.4 we prove that in the sub-Lorentzian geometry there exist non-Hamiltonian geodesics which are regular curves.

1.2. Basic definitions and facts on sub-Lorentzian geometry. See [6] for all details. Fix a sub-Lorentzian manifold $(M, H, h)$. For each point $p \in M$ a vector $v \in H_{p}$ is called horizontal. An absolutely continuous curve which is tangent to $H$ a.e. and has square integrable derivative is called a horizontal (or admissible) curve.

Unless otherwise specified, all vectors and curves are supposed to be horizontal.

A vector $v \in H_{p}$ is called timelike if $h(v, v)<0$, spacelike if $h(v, v)>0$ or $v=0$, null if $h(v, v)=0$ and $v \neq 0$, nonspacelike if $h(v, v) \leq 0$. A curve is called timelike if its tangent is timelike almost everywhere; similarly for spacelike, null and nonspacelike curves.

By a time orientation of $(M, H, h)$ we mean a timelike vector field on $M$. From now on we suppose our $(M, H, h)$ to be time-oriented.

If $X$ is a time orientation, then a nonspacelike $v \in H_{p}$ is called future directed if $h(v, X(p))<0$, and is called past directed if $h(v, X(p))>0$.

Throughout this paper f.d. stands for "future directed", $t$. for "timelike", and $n s p c$. for "nonspacelike". So, for instance, a t.f.d. curve is a curve which is horizontal and timelike future directed. 
By $\mathcal{H}$ we will denote the Hamiltonian associated with our sub-Lorentzian metric. Locally it can be defined as follows: let $X_{0}, X_{1}, \ldots, X_{k}$ be an orthonormal frame for $H$ defined on an open $U$ with $X_{0}$ timelike; then

$$
\mathcal{H}(x, \lambda)=-\frac{1}{2}\left\langle\lambda, X_{0}\right\rangle^{2}+\frac{1}{2} \sum_{j=1}^{k}\left\langle\lambda, X_{j}\right\rangle^{2}
$$

on $T^{*} M \mid U$. By $\overrightarrow{\mathcal{H}}$ we denote the corresponding Hamiltonian vector field on $T^{*} M$, and $\Phi_{s}$ stands for its flow. Notice that $\pi \circ \Phi_{s}(\lambda)=\pi \circ \Phi_{1}(s \lambda)$ for any covector $\lambda$, where $\pi: T^{*} M \longrightarrow M$ is the canonical projection.

A curve $\gamma:[\alpha, \beta] \longrightarrow U$ is called a Hamiltonian geodesic if it is of the form $\gamma(s)=$ $\pi \circ \Phi_{s}(\lambda)$. Each Hamiltonian geodesic is either timelike, spacelike or null (i.e. it does not change its casual character).

For a $p \in M$ denote by $\exp _{p}$ the exponential mapping with the pole at $p \in M$, which is defined as follows. Let $D_{p}$ stand for the set of all covectors $\lambda \in T_{p}^{*} M$ such that the curve $s \mapsto \pi \circ \Phi_{s}(\lambda)$ is defined on the interval $[0,1]$. The set $D_{p}$ is open and $\exp _{p}: D_{p} \longrightarrow M$ acts by the formula $\exp _{p}(\lambda)=\pi \circ \Phi_{1}(\lambda)$.

A point $q \in M$ is said to be conjugate to a point $p \in M$ if there is a $\lambda \in T_{p}^{*} M$ such that $\exp _{p}(\lambda)=q$ and $d_{\lambda} \exp _{p}$ is singular. Then we say that $q$ is conjugate to $p$ along a geodesic $\gamma(t)=\exp _{p}(t \lambda)$.

By the future (resp. past) timelike conjugate locus of a point $p$ we mean the set of all points conjugate to $p$ along timelike future (resp. past) directed Hamiltonian geodesics; timelike conjugate locus of $p$ is the union of the timelike future and past conjugate loci of $p$. In the similar manner we define spacelike and (future and past) null conjugate loci. Finally, the conjugate locus of a point $p$ is the union of the timelike, null, and spacelike conjugate loci of $p$.

For a nspc. curve $\gamma:[\alpha, \beta] \longrightarrow M$ we define its length to be

$$
L(\gamma)=\int_{\alpha}^{\beta}|h(\dot{\gamma}, \dot{\gamma})|^{1 / 2} d t
$$

Fix an open set $U$; a nspc.f.d. curve $\gamma:[\alpha, \beta] \longrightarrow U$ is called a maximizer with respect to $U$, or a $U$-maximizer, for short, if it is the longest curve from $\gamma(\alpha)$ to $\gamma(\beta)$ among all nspc.f.d. curves contained in $U$ and joining $\gamma(\alpha)$ to $\gamma(\beta)$. We also use a name $U$-geodesic for a curve in $U$ whose each suitably short sub-arc is a $U$-maximizer (note that in [6] only timelike curves were used; this is because the condition (A) was assumed).

By a unique $U$-maximizer (or a unique maximizing $U$-geodesic) we mean a (nspc.f.d.) curve $\gamma:[\alpha, \beta] \longrightarrow U$ such that for each $t_{1}, t_{2} \in[\alpha, \beta]$ with $t_{1}<t_{2}$, the restriction $\gamma \mid\left[t_{1}, t_{2}\right]$ is the only $U$-maximizer between $\gamma\left(t_{1}\right)$ and $\gamma\left(t_{2}\right)$. It can be proved that if $\gamma:[\alpha, \beta] \longrightarrow M$ is a t.f.d. Hamiltonian geodesic then for each $t \in(\alpha, \beta)$ there is a neighbourhood $U$ of $\gamma(t)$ such that $\gamma \cap U$ is a unique $U$-maximizer.

If $\varphi: U \longrightarrow \mathbb{R}$ is a smooth function on an open $U$, then its horizontal gradient $\nabla_{H} \varphi$ is, by definition, a vector field on $U$ such that $\left(\partial_{v} \varphi\right)(p)=h\left(v, \nabla_{H} \varphi(p)\right)$ for any $v \in H_{p}$ and $p \in U$. If $\nabla_{H} \varphi$ is unit timelike past directed on $U$ then the trajectories of $-\nabla_{H} \varphi$ are unique $U$-maximizers (indeed, let $\dot{\gamma}=-\nabla_{H} \varphi, \gamma(0)=p, \gamma(l)=q$; take $\eta:[\alpha, \beta] \longrightarrow U$ 
to be an arbitrary nspc.f.d. curve with $\eta(\alpha)=p, \eta(\beta)=q$. Now

$$
\begin{aligned}
& L(\gamma)=\varphi(\gamma(l))-\varphi(\gamma(0))=\varphi(\eta(\beta))-\varphi(\eta(\alpha))=\int_{\alpha}^{\beta} \frac{d}{d s}(\varphi(\eta(s)) d s \\
& =\int_{\alpha}^{\beta} h\left(\dot{\eta}, \nabla_{H} \varphi\right) d s \geq \int_{\alpha}^{\beta}|h(\dot{\eta}, \dot{\eta})|^{1 / 2} d s=L(\eta)
\end{aligned}
$$

by the reverse Schwarz inequality, and $L(\gamma)=L(\eta)$ holds if and only if $\gamma$ and $\eta$ are equal up to a change of parameter. See also [3] for the classical case where $H=T M$ and $\left.\nabla_{H}=\nabla\right)$.

$d[U](\cdot, \cdot)$ will denote the sub-Lorentzian distance function relative to a set $U \subset M$, which is defined as follows. For $p, q \in U$ let $\Omega_{p, q}(U)$ be the set of all nspc.f.d. curves contained in $U$ and joining $p$ to $q$; then

$$
d[U](p, q)= \begin{cases}\sup \left\{L(\gamma): \gamma \in \Omega_{p, q}(U)\right\} & \text { in case } \Omega_{p, q}(U) \neq \emptyset \\ 0 & \text { in case } \Omega_{p, q}(U)=\emptyset\end{cases}
$$

For a general $U$ very little can be said about $d[U]$. However for a fixed point $p_{0}$ one can construct a family of certain special neighbourhoods called normal neighbourhoods. If $U$ is such a normal neighbourhood of $p_{0}$ then one can prove that $d[U]$ is finite, and the function $p \mapsto d[U]\left(p_{0}, p\right)$ is upper semicontinuous on $U$. It is also continuous along smooth timelike $U$-maximizers.

We need some notion of convergence of sequences of curves. Suppose that $\gamma_{\nu}, \gamma$ : $[a, b] \longrightarrow M, \nu=1,2, \ldots$, are curves in $M$; we say that $\left\{\gamma_{\nu}\right\}$ converges to $\gamma$ in the $C^{0}$ topology on curves if $\gamma_{\nu}(a) \longrightarrow \gamma(a), \gamma_{\nu}(b) \longrightarrow \gamma(b)$, and for each open $V$ containing $\gamma$ there is an integer $\Lambda$ such that $\gamma_{\nu} \subset V$ for all $\nu>\Lambda$. Now, let $U$ be a normal neighbourhood of $p_{0}$ and take a sequence $\gamma_{\nu}:[0, l] \longrightarrow U$ of nspc.f.d. curves such that $\gamma_{\nu}(0)=p_{0}$ and their endpoints $\gamma_{\nu}(l)$ tend to a point $p \in U$. Then it can be proved that there exists a subsequence $\left\{\gamma_{\nu_{i}}\right\}$ convergent in the $C^{0}$ topology to a nspc.f.d. curve joining $p_{0}$ to $p$ and contained in $U$.

Remark that the definition of $d[U]$ and $U$-maximizers adopted in this paper is extended in comparison with [6] - we use nspc.f.d. curves instead of timelike; however the proofs presented in [6] remain still true.

At the end we introduce a notion of so-called regular curves. Fix a $p \in M$, and denote by $\Omega_{p}$ the set of all horizontal curves $\gamma:[0,1] \longrightarrow M$ starting from $\gamma(0)=p$. The endpoint map end en $_{p} \longrightarrow M$ is the mapping that assigns to each curve $\gamma \in \Omega_{p}$ its end $\gamma(1)$. $\operatorname{end}_{p}$ is of class $C^{\infty}$ with respect to the structure of Hilbert manifold on $\Omega_{p}$. Now, a curve $\gamma \in \Omega_{p}$ is said to be regular (resp. singular) if it is a regular (resp. critical) point of end . It can be proved that in a contact case only constant curves are singular.

\section{Heisenberg sub-Lorentzian metric}

2.1. Geodesics. From now on $M=\mathbb{R}^{3}$ and $(H, h)$ is the Heisenberg sub-Lorentzian metric defined above.

The Hamiltonian associated to our metric is of the form

$$
\mathcal{H}=-\frac{1}{2} f^{2}+\frac{1}{2} g^{2}
$$


where the functions $f, g: T^{*} \mathbb{R}^{3} \longrightarrow \mathbb{R}$ are defined by the formulae

$$
f=\langle\lambda, X\rangle, \quad g=\langle\lambda, Y\rangle,
$$

$\lambda \in T^{*} \mathbb{R}^{3}$. In other words

$$
f(x, y, z, p, q, r)=p+y r / 2, \quad g(x, y, z, p, q, r)=q-x r / 2,
$$

where $x, y, z$ are standard coordinates on $\mathbb{R}^{3}$ and $p, q, r$ are dual coordinates. Now one can easily calculate t.f.d. Hamiltonian geodesics starting from the origin. Considering the Hamiltonian equations with the function $\mathcal{H}$, first of all we see that $r(s) \equiv r(0)=r_{0}$. Next, the equations of geodesics take the form

$$
\dot{x}=-f, \quad \dot{y}=g, \quad \dot{z}=(y \dot{x}-x \dot{y}) / 2,
$$

where

$$
\dot{f}=r_{0} g, \quad \dot{g}=r_{0} f
$$

and the equation for $\dot{z}$ simply means horizontality of the solution curves. Since we are looking for future directed geodesics parametrized by arc-length, we take initial conditions from the set $C^{-}$:

$$
C^{-}=\left\{\left(0,0,0,-\cosh \varphi, \sinh \varphi, r_{0}\right): \varphi, r_{0} \in \mathbb{R}\right\} \subset T_{0}^{*} \mathbb{R}^{3}
$$

Solving our equations we get

$$
\left\{\begin{array}{l}
x(s)=\frac{2}{r_{0}} \cosh \left(\varphi-\frac{r_{0} s}{2}\right) \sinh \left(\frac{r_{0} s}{2}\right) \\
y(s)=\frac{2}{r_{0}} \sinh \left(\varphi-\frac{r_{0} s}{2}\right) \sinh \left(\frac{r_{0} s}{2}\right) \\
z(s)=-\frac{1}{2 r_{0}^{2}}\left(r_{0} s-\sinh \left(r_{0} s\right)\right),
\end{array}\right.
$$

for $r_{0} \neq 0$, and

$$
\left\{\begin{array}{l}
x(s)=s \cosh \varphi \\
y(s)=s \sinh \varphi \\
z(s)=0
\end{array}\right.
$$

for $r_{0}=0$.

Looking at (2.1) and (2.2) one can see that projections on the $(x, y)$-plane of t.f.d. Hamiltonian geodesics emanating from zero are either half-lines or segments of hyperbolas contained in the set

$$
Z=\{(x, y, 0):|y|<|x|, x>0\} .
$$

It should be noted here that timelike Hamiltonian geodesics are the only timelike geodesics, as can be seen from the Pontryagin Maximum Principle applied to our case.

Next, in a similar way we calculate null and spacelike Hamiltonian geodesics. Thus we find that there are only two null f.d. geodesics, namely half-lines $y= \pm x, x>0$, in the plane $\{z=0\}$, and that, for instance, spacelike geodesics with initial conditions from 
the set $\left\{\left(0,0,0, \sinh \varphi, \cosh \varphi, r_{0}\right)\right\}$ are of the form

$$
\left\{\begin{array}{l}
x(s)=-\frac{2}{r_{0}} \sinh \left(\varphi+\frac{r_{0} s}{2}\right) \sinh \frac{r_{0} s}{2} \\
y(s)=\frac{2}{r_{0}} \cosh \left(\varphi+\frac{r_{0} s}{2}\right) \sinh \frac{r_{0} s}{2} \\
z(s)=\frac{1}{2 r_{0}^{2}}\left(r_{0} s-\sinh \left(r_{0} s\right)\right) .
\end{array}\right.
$$

In particular no timelike nor spacelike Hamiltonian geodesic intersects the set $\{(x, y): y= \pm x\} \times \mathbb{R}$. In this way we obtain

\section{Corollary 2.1. For the Heisenberg sub-Lorentzian metric}

$$
0 \notin \operatorname{Int}\left(\exp _{0}\left(D_{0}\right)\right) \text {. }
$$

Remark that such a situation is impossible in the contact sub-Riemannian case; it is well known that if we take a point $p$ on a contact sub-Riemannian manifold then the exponential mapping $\exp _{p}$ is onto a neighbourhood of $p$ (since one can find a neighbourhood $V$ of $p$ such that each $q \in V$ can be joined to $p$ by a Hamiltonian geodesic contained in $V)$.

2.2. Exponential mapping and the conjugate locus. We will compute the conjugate locus of 0 . To this end we must find singularities of $\exp _{0}: T_{0}^{*} \mathbb{R}^{3} \longrightarrow \mathbb{R}^{3}$. In order to find the future timelike conjugate locus, we consider the exponential mapping as the mapping

$$
\mathcal{E}:(0, \infty) \times \mathbb{R} \times \mathbb{R} \longrightarrow \mathbb{R}^{3},
$$

defined by the formula

$$
\mathcal{E}\left(s, \varphi, r_{0}\right)=\exp _{0} s\left(-\cosh \varphi, \sinh \varphi, r_{0}\right)=\pi \circ \Phi_{s}\left(0,0,0,-\cosh \varphi, \sinh \varphi, r_{0}\right) ;
$$

here $\left(s, \varphi, r_{0}\right) \approx s\left(-\cosh \varphi, \sinh \varphi, r_{0}\right)$ can be regarded as coordinates on a subset of $T_{0}^{*} \mathbb{R}^{3}$ which is diffeomorphic to $(0, \infty) \times C^{-}$. Now one can see that

$$
\operatorname{det}\left(d \mathcal{E}\left(s, \varphi, r_{0}\right)\right)=\frac{4}{r_{0}^{4}} \sinh \frac{r_{0} s}{2}\left(\sinh \frac{r_{0} s}{2}-\frac{r_{0} s}{2} \cosh \frac{r_{0} s}{2}\right)
$$

for $r_{0} \neq 0$, and

$$
\operatorname{det}(d \mathcal{E}(s, \varphi, 0))=-\frac{1}{12} s^{4}
$$

for $r_{0}=0$. Clearly $\operatorname{det}\left(d \mathcal{E}\left(s, \varphi, r_{0}\right)\right)$ vanishes nowhere for $s>0$; it means that the future conjugate locus of 0 is a single point 0 . Similar calculations show that the same result holds for the past timelike and spacelike conjugate locus. On the other hand, it is obvious that $\exp _{0}$ must be degenerate on the set

$$
\left\{\left(0,0,0, \lambda, \pm \lambda, r_{0}\right): \lambda \neq 0, r_{0} \in \mathbb{R}\right\} .
$$

In this way we have obtained

Proposition 2.1. For the Heisenberg sub-Lorentzian metric on $\mathbb{R}^{3}$ the timelike and spacelike conjugate loci of the origin degenerate to the point 0 . It follows that the conjugate locus coincides with the null conjugate locus, and the latter is formed by the two null 
Hamiltonian geodesics passing through 0 . Moreover, the restriction of $\mathcal{E}$ to a neighbourhood $V \subset T_{0}^{*} \mathbb{R}^{3}$ of the set $(0, \infty) \times \mathbb{R} \times\{0\}$ is a diffeomorphism onto a neighbourhood $W \subset \mathbb{R}^{3}$ of the set $Z$.

Proof. The last statement follows from [4]: the restriction

$$
\mathcal{E}:(0, \infty) \times \mathbb{R} \times\{0\} \longrightarrow Z
$$

is a diffeomorphism and $d \mathcal{E}$ is non-singular on $(0, \infty) \times \mathbb{R} \times\{0\}$.

2.3. Properties of the distance function. Proposition 2.1 has a few easy interesting consequences. Let $V$ and $W$ be as in Proposition 2.1 , so that $\left(s, \varphi, r_{0}\right)$ can be regarded as coordinates on $W$.

First of all let us notice that $\partial / \partial s$ is a unit t.f.d. vector field on $W$. Indeed, a curve $\gamma(s)=\exp _{0} s\left(-\cosh \varphi, \sinh \varphi, r_{0}\right)$ is a t.f.d. geodesic which is parametrized by arc-length. In our coordinates $\gamma(s)=\left(s, \varphi, r_{0}\right)$, from which it follows that $\dot{\gamma}(s)=(\partial / \partial s)_{\gamma(s)}$.

Next, let $\xi: W \longrightarrow \mathbb{R}$ be a function defined by $\xi(P)=s$, where $\mathcal{E}\left(s, \varphi, r_{0}\right)=P$ and $\left(s, \varphi, r_{0}\right) \in V$. We will show that

$$
\nabla_{H} \xi=-\partial / \partial s
$$

Let $X=\partial / \partial s, Y$ be an orthonormal basis of $H$ over $W$. Then of course

$$
\nabla_{H} \xi=-\left(\partial_{X} \xi\right) X+\left(\partial_{Y} \xi\right) Y=-\partial / \partial s+\left(\partial_{Y} \xi\right) Y
$$

We will show that $Y$ can be chosen in the form

$$
Y=A \partial / \partial \varphi+B \partial / \partial r_{0}
$$

for some smooth function $A$ and $B$. To this end denote by $\left(\bar{s}, \bar{\varphi}, \bar{r}_{0}\right)$ the corresponding coordinates on $V \subset T_{0}^{*} \mathbb{R}^{3}$; in other words we have

$$
\frac{\partial}{\partial \varphi}=\left(\pi \circ \Phi_{s}\right)_{*} \frac{\partial}{\partial \bar{\varphi}}, \quad \frac{\partial}{\partial r_{0}}=\left(\pi \circ \Phi_{s}\right)_{*} \frac{\partial}{\partial \bar{r}_{0}} .
$$

Take $\lambda$ of the form

$$
\left(0,0,0,-\cosh \varphi, \sinh \varphi, r_{0}\right) .
$$

Let $\alpha$ stand for the Liouville form on $T^{*} \mathbb{R}^{3}$. Note that our Hamiltonian $\mathcal{H}$ is homogeneous in $p, q, r$ so the flow $\Phi_{s}$ preserves the form $\alpha$ restricted to level surfaces of $\mathcal{H}$. Thus we obtain, using the definition of $\alpha$

$$
\left\langle\Phi_{s}(\lambda), \frac{\partial}{\partial \varphi}\right\rangle=\left\langle\alpha\left(\Phi_{s}(\lambda)\right), \Phi_{s *} \frac{\partial}{\partial \bar{\varphi}}\right\rangle=\left\langle\lambda, \pi_{*} \frac{\partial}{\partial \bar{\varphi}}\right\rangle=0 .
$$

Similarly we obtain

$$
\left\langle\Phi_{s}(\lambda), \frac{\partial}{\partial r_{0}}\right\rangle=0
$$

Now take a $P \in W ; P=\gamma(s)=\exp _{0}(s \lambda)$, where $\lambda$ is as in (2.4). Then

$$
h(X, Y)_{\gamma(s)}=A\left\langle\Phi_{s}(\lambda), \frac{\partial}{\partial \varphi}\right\rangle+B\left\langle\Phi_{s}(\lambda), \frac{\partial}{\partial r_{0}}\right\rangle=0
$$

from which $\partial_{Y} \xi=0$ and the proof of (2.3) is over. It follows that $\nabla_{H} \xi$ is unit timelike past directed. 
Let

$$
s_{0}\left(\varphi, r_{0}\right)=\sup \left\{s: \exp _{0}\left(\sigma, \varphi, r_{0}\right) \in W \text { for all } \sigma \in(0, s)\right\} ;
$$

for instance $s_{0}(\varphi, 0)=\infty$. Now t.f.d. Hamiltonian geodesics of the form

$$
s \longrightarrow \exp _{0}\left(s, \varphi, r_{0}\right), 0<s<s_{0}\left(\varphi, r_{0}\right),
$$

are trajectories of $-\nabla_{H} \xi$.

We will need the following

LEMMA 2.1. For any normal neighbourhood $U$ of 0 , the half-lines

$$
y=a x, \quad z=0, \quad-1<a<1
$$

are unique $U$-maximizers.

Proof. It suffices to notice that the curves in (2.6) are trajectories of $-\nabla_{H} \varphi$, where $\varphi(x, y, z)=\left(x^{2}-y^{2}\right)^{1 / 2},|y|<|x|$.

Fix a normal neighbourhood $U$ of 0 and set

$$
f[U](P)=d[U](0, P) .
$$

We may suppose that $W \subset U$. We will show that there exists an open $W_{0}$ with $0 \in \partial W_{0}$, $Z \subset W_{0} \subset W$, and such that for each $P \in W_{0}$ there is a $U$-maximizer joining 0 to $P$, which is contained in $W \cup\{0\}$. Suppose that this is not the case. Then for a point $P_{0} \in Z$ we can find a sequence $P_{\nu} \longrightarrow P_{0}$ with the property that there is a $U$-maximizer $\gamma_{\nu}$ joining 0 to $P_{\nu}$, and such that $\gamma_{\nu} \backslash(W \cup\{0\}) \neq \emptyset$. Passing to a subsequence we may suppose that $\gamma_{\nu} \longrightarrow \gamma$ in the $C^{0}$-topology on curves, where $\gamma$ is nspc.f.d., connects 0 to $P_{0}$, and $\gamma \backslash(W \cup\{0\}) \neq \emptyset$. But

$$
f[U]\left(P_{0}\right)=\lim _{\nu \longrightarrow \infty} f[U]\left(P_{\nu}\right)=\lim _{\nu \longrightarrow \infty} L\left(\gamma_{\nu}\right) \leq L(\gamma)
$$

by upper semicontinuity of sub-Lorentzian arc-length, so $\gamma$ is a $U$-maximizer, and hence (Lemma 2.1) is of the form (2.6). In particular, $\gamma \subset W \cup\{0\}$ which gives a contradiction.

Now it follows that $f[U]$ coincides with $\xi$ on $W_{0}$. In this way we have established

Proposition 2.2. Let $U$ be a normal neighbourhood of 0 . There exists an open $W_{0} \subset U, 0 \in \partial W_{0}, Z \subset W_{0}$, such that t.f.d. Hamiltonian geodesics of the form (2.5) which are contained in $W_{0}$ are unique $U$-maximizers. Moreover the Heisenberg sub-Lorentzian distance from the origin with respect to $U, f[U]$, is smooth on $W_{0}$.

2.4. Existence of regular non-Hamiltonian geodesics. In this subsection we describe certain phenomena which do not occur in the sub-Riemannian case. It is a simple consequence of the above results.

Proposition 2.3. Let $U$ be a normal neighbourhood of 0 . Then there exists a nonHamiltonian $U$-maximizer which is a regular curve.

Proof. Consider the set $B=\exp _{0}\left(B_{0}\right) \cap U$, where

$$
B_{0}=\left\{\left(0,0,0,-R \cosh \varphi, R \sinh \varphi, r_{0}\right): \varphi, r_{0} \in \mathbb{R}, R>0\right\} \subset T_{0}^{*} \mathbb{R}^{3} .
$$

Evidently, $B$ is an open subset in $\mathbb{R}^{3}$, as it follows from Proposition 2.1. Take a point $P \in \partial B$ which lies neither on $\partial U$ nor on any of the two null Hamiltonian geodesics starting 
from 0 . Take a sequence $B \ni P_{\nu} \longrightarrow P$. From the properties of normal neighbourhoods (cf. Proposition 5.3 in [6]) we know that for each $\nu$ there exists a $U$-maximizer $\gamma_{\nu}$ joining 0 to $P_{\nu}$. After passing to a subsequence we have $\gamma_{\nu} \longrightarrow \gamma$ in the $C^{0}$ topology on curves, where $\gamma$ is nspc.f.d. and connects 0 to $P$. It follows that there exists a $U$-maximizer joining 0 to $P$, which is regular (since our distribution is contact), and clearly cannot be Hamiltonian.

Acknowledgements. This research was partially supported by EC IHP programme contract number HPRN-CT-00271.

\section{References}

[1] A. A. Agrachev, El-H. Chakir El-Alaoui, J.-P. Gauthier, Sub-Riemannian metrics on $\mathbb{R}^{3}$, in: Geometric Control and Non-holonomic Mechanics (Mexico City, 1996), CMS Conf. Proc. 25, Amer. Math. Soc., Providence, 1998, 29-78.

[2] El-H. Ch. El-Alaoui, J.-P. Gauthier, I. Kupka, Small sub-Riemannian balls in $\mathbb{R}^{3}$, J. Dynam. Control Systems 2 (1996), 359-421.

[3] J. K. Beem, P. E. Ehrlich, K. L. Easley, Global Lorentzian Geometry, Marcel Dekker, New York, 1996.

[4] M. Golubitsky, V. Guillemin, Stable Mappings and their Singularities, Graduate Texts in Math. 14, Springer, New York, 1973.

[5] M. Grochowski, Differential properties of the sub-Riemannian distance function, Bull. Polish Acad. Sci. Math. 50 (2002), 93-101.

[6] M. Grochowski, Geodesics in the sub-Lorentzian geometry, Bull. Polish Acad. Sci. Math. 50 (2002), 161-178. 